\title{
COMPARATOR IDENTIFICATION IN THE CONDITIONS OF BIFUZZY INITIAL DATA
}

\author{
Lev Raskin \\ Department of Distributed information systems and cloud technologies ${ }^{1}$ \\ Oksana Sira \\ Department of Distributed information systems and cloud technologies ${ }^{1}$ \\ topology@ukr.net \\ Tetiana Katkova \\ Department of Cyber security \\ University of Customs and Finance \\ 2/4 V. Vernadsky str., Dnipro, Ukraine, 49000 \\ 777-kit@ukr.net \\ ${ }^{1}$ National Technical University «Kharkiv Polytechnic Institute» \\ 2 Kyrpychova str., Kharkiv, Ukraine, 61002
}

\begin{abstract}
When solving a large number of problems in the study of complex systems, it becomes necessary to establish a relationship between a variable that sets the level of efficiency of the system's functioning and a set of other variables that determine the state of the system or the conditions of its operation. To solve this problem, the methods of regression analysis are traditionally used, the application of which in many real situations turns out to be impossible due to the lack of the possibility of direct measurement of the explained variable. However, if the totality of the results of the experiments performed can be ranked, for example, in descending order, thus forming a system of inequalities, the problem can be presented in such a way as to determine the coefficients of the regression equation in accordance with the following requirement. It is necessary that the results of calculating the explained variable using the resulting regression equation satisfy the formed system of inequalities. This task is called the comparator identification task.

The paper proposes a method for solving the problem of comparator identification in conditions of fuzzy initial data. A mathematical model is introduced to describe the membership functions of fuzzy parameters of the problem based on functions $(L-R)$-type. The problem is reduced to a system of linear algebraic equations with fuzzy variables.

The analytical relationships required for the formation of a quality criterion for solving the problem of comparator identification in conditions of fuzzy initial data are obtained. As a result, a criterion for the effectiveness of the solution is proposed, based on the calculation of membership functions of the results of experiments, and the transformation of the problem to a standard problem of linear programming is shown. The desired result is achieved by solving a quadratic mathematical programming problem with a linear constraint. The proposed method is generalized to the case when the fuzzy initial data are given bifuzzy.
\end{abstract}

Keywords: regression analysis, comparator identification problem, fuzzy and bifuzzy values of the initial data.

DOI: $10.21303 / 2461-4262.2021 .001609$

\section{Introduction}

In the process of studying complex systems of various natures, it becomes necessary to build a mathematical model in the form of a regression equation. Such equations set the level of efficiency of the system operation depending on the variables that determine the state of the system or the conditions of its operation. Traditionally, the method of least squares is used to calculate the coefficients of the regression model, and the task may be to determine the coefficients of the regression equation in such a way that the results of calculating the explained variable using the resulting regression equation satisfy the formed system of inequalities. This task is called the comparator identification task.

The problem of comparator identification is a special, non-template version of regression analysis $[1,2]$, which arises in the following case. Let's suppose that as a result of a series of experiments in $j$-th of them, a set of values of factors (explanatory variables) $F_{j 1}, F_{j 2}, \ldots, F_{j m}$ is obtained influencing the outcome of the experiment, and the value of the explained variable in this experiment $-y_{j}, j=1,2, \ldots, n$. The resulting array of initial data is jointly processed in order to find a set of unknown coefficients $x_{0}, x_{1}, \ldots, x_{m}$ that define the regression equation: 


$$
y_{j}=x_{0}+x_{1} F_{j 1}+x_{2} F_{j 2}+\ldots+x_{m} F_{j m}, \quad j=1,2, \ldots, n
$$

determining the dependence of the explained variable on the explanatory ones. The required set $X=\left(x_{0}, x_{1}, \ldots, x_{m}\right)$ is found by the least squares method using the formula:

where

$$
X=\left(H^{T} H\right)^{-1} H^{T} Y,
$$

$$
H=\left(\begin{array}{ccccc}
1 & F_{11} & F_{12} & \ldots & F_{1 m} \\
1 & F_{21} & F_{22} & \ldots & F_{2 m} \\
\ldots & \ldots & \ldots & \ldots & \ldots \\
1 & F_{n 1} & F_{n 2} & \ldots & F_{n m}
\end{array}\right), \quad X=\left(\begin{array}{l}
x_{0} \\
x_{1} \\
\ldots \\
x_{m}
\end{array}\right), \quad Y=\left(\begin{array}{l}
y_{1} \\
y_{2} \\
\ldots \\
y_{n}
\end{array}\right) .
$$

Here $F_{j i}$ - value of the $i$-th factor in the $j$-th experiment.

Let now, for some reason, the value of the explained variable in each experiment can't be determined, but they can be ranked, for example, in ascending order. Then (after renumbering) let's obtain a chain of inequalities:

$$
y_{1}<y_{2}<\ldots<y_{n}
$$

Now the task is to find the vector $X$, which, taking into account (1), ensures the fulfillment of inequalities (3). The problem formulated in this way is called the comparator identification problem [3]. The following method for solving this problem is known [4-8]. Using (3), taking into account (1), let's form the following system of inequalities:

$$
\begin{aligned}
& y_{1}-y_{2}=x_{1}\left(F_{11}-F_{21}\right)+x_{2}\left(F_{12}-F_{22}\right)+\ldots+x_{m}\left(F_{1 m}-F_{2 m}\right)<0 \text {, } \\
& y_{2}-y_{3}=x_{1}\left(F_{21}-F_{31}\right)+x_{2}\left(F_{22}-F_{32}\right)+\ldots+x_{m}\left(F_{2 m}-F_{3 m}\right)<0 \text {, } \\
& y_{n-1}-y_{n}=x_{1}\left(F_{n-1,1}-F_{n 1}\right)+x_{2}\left(F_{n-1,2}-F_{n 2}\right)+\ldots+x_{m}\left(F_{n-1, m}-F_{n m}\right)<0 \text {. }
\end{aligned}
$$

System of inequalities (4) by adding positive $x_{m+1}, x_{m+2}, \ldots, x_{m+n-1}$ is transformed to the system of equalities:

$$
\begin{gathered}
\sum_{i=1}^{m} x_{i} r_{j i}+x_{m+j}=0, \quad j=1,2, \ldots, n-1, \\
x_{i} \geq 0, \quad x_{m+j}>0, \\
r_{j i}=F_{j i}-F_{j+1, i}, \quad i=1,2, \ldots, m, \quad j=1,2, \ldots, n-1 .
\end{gathered}
$$

The problem is to find a set of variables $x_{0}, x_{1}, \ldots, x_{m}, x_{m+1}, x_{m+2}, \ldots, x_{m+n-1}$, satisfying equations (5). In order to eliminate the trivial zero solution to the problem $\left(x_{i}=0, i=1,2, \ldots, m+n-1\right)$, let's add one more constraint:

$$
\sum_{i=1}^{m} x_{i}+\sum_{j=1}^{n-1} x_{m+j}=1 .
$$

The resulting system of linear algebraic equations (5), (6) is redefined and, possibly, inconsistent. Therefore, a natural approach to solving it is to minimize the quadratic form:

$$
L(X)=\sum_{j=1}^{n-1}\left(\sum_{i=1}^{m} x_{i} r_{j i}+x_{m+j}\right)^{2}
$$

Taking into account the constraint (6).

Let's solve the formulated problem of mathematical programming by the method of indefinite Lagrange multipliers. The Lagrange function has the form. 


$$
\Phi(X)=\sum_{j=1}^{n-1}\left(\sum_{i=1}^{m} x_{i} r_{j i}+x_{m+j}\right)^{2}-\lambda\left(\sum_{i=1}^{m} x_{i}+\sum_{j=1}^{n-1} x_{m+j}-1\right) .
$$

So,

$$
\begin{gathered}
\frac{d \Phi(X)}{d x_{k}}=2 \sum_{j=1}^{n-1}\left(\sum_{i=1}^{m} x_{i} r_{j i}+x_{m+j}\right) r_{j k}-\lambda=2\left[\sum_{i=1}^{m} x_{i} \sum_{j=1}^{n-1} r_{j i} r_{j k}+\sum_{j=1}^{n-1} x_{m+j} \cdot r_{j k}\right]-\lambda= \\
=2 \sum_{i=1}^{m} x_{i} c_{k i}+2 \sum_{j=1}^{n-1} x_{m+j} \cdot r_{j k}-\lambda=0, \\
c_{k i}=\sum_{j=1}^{n-1} r_{j i} \cdot r_{j k}, \quad k=1,2, \ldots, m .
\end{gathered}
$$

Hence,

$$
\sum_{i=1}^{m} x_{i} c_{k i}+\sum_{j=1}^{n-1} x_{m+j} \cdot r_{j k}=\frac{\lambda}{2}, \quad k=1,2, \ldots, m
$$

Further,

$$
\frac{d \Phi}{d x_{m+j}}=2\left(\sum_{i=1}^{m} x_{i} r_{j i}+x_{m+j}\right)-\lambda=0
$$

hence,

$$
\sum_{i=1}^{m} x_{i} r_{j i}+x_{m+j}=\frac{\lambda}{2}, \quad j=1,2, \ldots, n-1 .
$$

Relations (9) and (10) form a system of linear algebraic equations containing $m+n-1$ equations and the same number of unknowns. Let's introduce the matrix $M$ of coefficients in front of the unknowns, the vector column of unknowns $X^{T}=\left(x_{1}, \ldots, x_{m}, x_{m+1}, \ldots, x_{m+n-1}\right)$, as well as the column of free terms $\Lambda^{T}=1 / 2(\lambda, \lambda, \ldots, \lambda), \operatorname{dim} \Lambda=(n-1) \times 1$. Then the control system (9), (10) in matrix form has the form:

$$
M X=\Lambda .
$$

Hence,

$$
X=M^{-1} \Lambda
$$

Using (9) and (10), let's consider the structure of the matrix $M$.

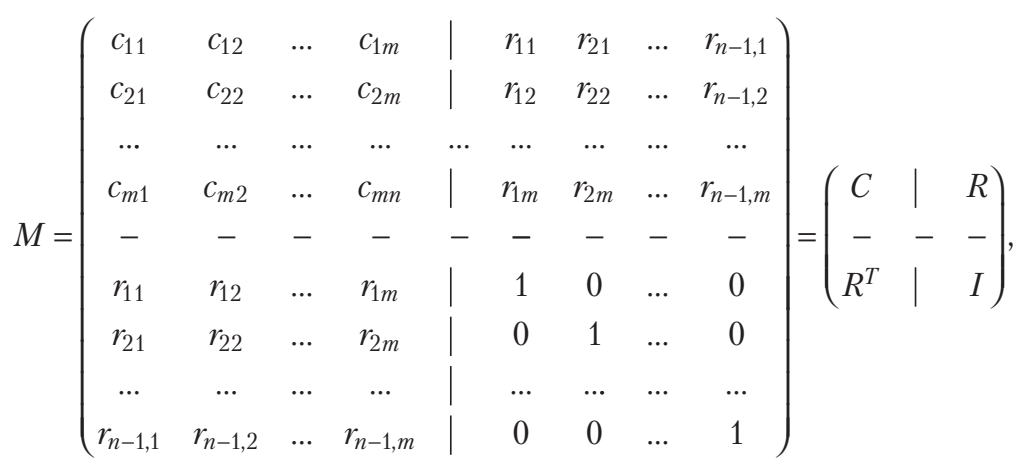

$$
\begin{aligned}
& \operatorname{dim} C=m \times m, \quad \operatorname{dim} R=m \times(n-1), \operatorname{dim} I=(n-1) \times(n-1), \\
& \operatorname{dim} M=(m+n-1) \times(m+n-1)=Q \times Q .
\end{aligned}
$$


The block character of the matrix $M$ allows to use the Frobenius formula [9]:

$$
\begin{gathered}
M^{-1}=\left(\begin{array}{ll}
M_{1} & M_{2} \\
M_{3} & M_{4}
\end{array}\right)^{-1}=\left(\begin{array}{c|c}
M_{1}^{-1}+M_{1}^{-1} M_{2} H^{-1} M_{3} M_{1}^{-1} & -M_{1}^{-1} M_{2} H^{-1} \\
------------- & --------- \\
-H^{-1} M_{3} M_{1}^{-1} & H^{-1}
\end{array}\right), \\
H=M_{4}-M_{3} M_{1}^{-1} M_{2} .
\end{gathered}
$$

Let's rewrite this formula taking into account that:

$$
M_{1}=C, \quad M_{2}=R, \quad M_{3}=R^{T}, \quad M_{4}=I .
$$

Thus let's obtain:

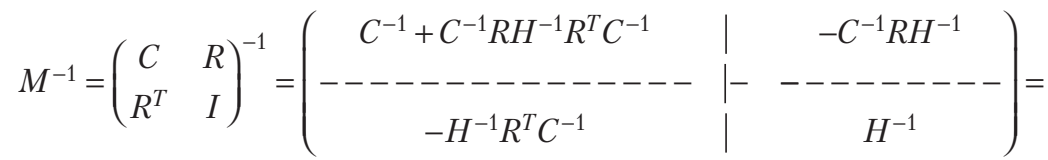

$$
\begin{aligned}
& =\left(\begin{array}{cccccc}
m_{11} & m_{12} & \ldots & m_{1 q} & \ldots & m_{1 Q} \\
m_{21} & m_{22} & \ldots & m_{2 q} & \ldots & m_{2 Q} \\
\ldots & \ldots & \ldots & \ldots & \ldots & \ldots \\
m_{q 1} & m_{q 2} & \ldots & m_{q q} & \ldots & m_{q Q} \\
\ldots & \ldots & \ldots & \ldots & \ldots & \ldots \\
m_{Q 1} & m_{Q 2} & \ldots & m_{Q q} & \ldots & m_{Q Q}
\end{array}\right), \\
& H=I-R^{T} A^{-1} R .
\end{aligned}
$$

Since the dimensions of the matrices $C$ and are less than the dimension of the matrix $M$, the block inversion of the matrix $M$ simplifies the procedure for obtaining a set of dependences of the values of the variables $x_{q}$ on $\lambda, q=1,2, \ldots, Q, Q=m+n-1$.

Then from (11):

$$
X=\left(\begin{array}{l}
x_{0} \\
x_{1} \\
\ldots \\
x_{Q}
\end{array}\right)=\frac{1}{2}\left(\begin{array}{cccc}
m_{11} & m_{12} & \ldots & m_{1 Q} \\
m_{21} & m_{22} & \ldots & m_{2 Q} \\
\ldots & \ldots & \ldots & \ldots \\
m_{Q 1} & m_{Q 2} & \ldots & m_{Q Q}
\end{array}\right) \cdot\left(\begin{array}{l}
\lambda \\
\lambda \\
\ldots \\
\lambda
\end{array}\right)=\frac{\lambda}{2}\left(\begin{array}{cccc}
m_{11} & m_{12} & \ldots & m_{1 Q} \\
m_{21} & m_{22} & \ldots & m_{2 Q} \\
\ldots & \ldots & \ldots & \ldots \\
m_{Q 1} & m_{Q 2} & \ldots & m_{Q Q}
\end{array}\right) \cdot\left(\begin{array}{c}
1 \\
1 \\
\ldots \\
1
\end{array}\right) .
$$

Hence,

$$
x_{s}=\frac{\lambda}{2} \sum_{q=1}^{Q} m_{s q}, \quad s=1,2, \ldots, Q .
$$

Now, using (6), let's find the value $\lambda$ :

$$
\sum_{s=1}^{Q} x_{s}=\frac{\lambda}{2} \sum_{s=1}^{Q} \sum_{q=1}^{Q} m_{s q}=1 \text {. }
$$

Hence,

$$
\frac{\lambda}{2}=\frac{1}{\sum_{s=1}^{Q} \sum_{q=1}^{Q} m_{s q}} .
$$


Then

$$
x_{s}=\frac{\sum_{q=1}^{Q} m_{s q}}{\sum_{s=1}^{Q} \sum_{q=1}^{Q} m_{s q}}, s=1,2, \ldots, Q .
$$

The solution to the problem is obtained. However, it should be noted that the problem of comparator identification becomes much more complicated if its initial data are not accurate. Let, for example, the values of factors in a series of experiments are described unclearly [10]. In this case, let's assume that on the basis of all available information, membership functions of the corresponding fuzzy numbers can be constructed. It is clear that the method described above for solving the standard problem of comparator identification under these conditions can't be used. The indistinctness of the initial data gives rise to the indistinctness of the result. Based on this, it should be recognized that the research is relevant, the results of which allow to solve this problem.

The paper proposes a method for solving the problem of comparator identification in conditions of fuzzy initial data. A mathematical model is introduced to describe the membership functions of fuzzy parameters of the problem based on functions $(L-R)$-type. The problem is reduced to a system of linear algebraic equations with fuzzy variables.

The desired result is achieved by solving a quadratic mathematical programming problem with a linear constraint. The proposed method is generalized to the case when the fuzzy initial data are given bifuzzy.

\section{The aim and objectives of research}

The aim of research is to develop a method for solving the problem of comparator identification with indistinctly specified initial data.

To achieve the aim, the following objectives must be solved:

1. Formulate the correct formulation of the comparator identification problem under conditions when the uncertainty of the initial data is described in terms of fuzzy mathematics.

2. Justify the quality criterion for solving the comparator identification problem with fuzzy initial data.

3. Develop a procedure for solving the problem of comparator identification when choosing fuzzy numbers $(L-R)$-type as input data.

\section{Results of developing a method for solving the problem}

\section{1. Problem statement}

Let a series of experiments be carried out, in each of which the values of influencing factors are fuzzy numbers with known membership functions $\mu\left(F_{j i}\right), j=1,2, \ldots, n, i=1,2, \ldots, m$. Let, in addition, the unknown values of the explained variable can be ranged, as a result of which a system of inequalities (3) is obtained. It is required to find a vector $X$ that provides the calculation of a set of values of the explained variable, which satisfies (3) and optimizes the selected criterion.

Let's consider the following scheme for solving the problem of comparator identification. First, let's choose the type of analytical description of the membership functions of fuzzy initial data. Then let's construct a mathematical model of the problem, on the basis of which let's formulate a naturally interpreted and easily computable criterion for the quality of the solution to the identification problem. At the end of the solution, let's develop a method for optimizing the selected criterion.

\section{2. Justification of the quality criterion for solving the problem of comparator identi-} fication with fuzzy initial data

In the practice of solving various problems of fuzzy mathematics, an extensive set of types of membership functions of fuzzy numbers is used, for example, triangular, trapezoidal, Gaussian and many others. At the same time, recently more and more often the method of constructing 
membership functions is used, based on the approximation of known types of membership functions by so-called functions $(L-R)$-type. The attractiveness of using membership functions $(L-R)$-type is explained by two important properties. First, these functions are easily and conveniently defined by a fixed set of values of numeric parameters. Secondly, in relation to numbers with a membership function $(L-R)$-type, a strict system of rules for performing algebraic operations has been developed [11]. To implement these rules, it is necessary and sufficient to know the values of the sets of numerical values of the parameters of the membership functions of fuzzy numbers - the operands. At the same time, in most practical problems, it is acceptable to use three-parameter membership functions $(L-R)$ - of the type, having the form:

$$
\mu(x)=\left\{\begin{array}{l}
0, x<m-\alpha \\
L\left(\frac{m-x}{\alpha}\right), m-\alpha \leq x<m \\
R\left(\frac{x-m}{\beta}\right), m \leq x \leq m+\beta \\
0, x>m+\beta .
\end{array}\right.
$$

Here $m$ - modal value of the fuzzy number $x, \alpha$ - left fuzzy factor of the number $x, \beta$-right fuzzy factor of the number $x$.

Functions $L((m-x) / \alpha)$ and $R((x-m) / \beta)$ are arbitrary monotone functions for which:

$$
L: \mathbf{R} \rightarrow[0 ; 1], R: \mathbf{R} \rightarrow[0 ; 1], L(0)=R(0)=1 .
$$

In what follows, using the generally accepted notation, to describe three-parameter fuzzy numbers $(L-R)$-type, instead of the cumbersome relation (12), let's use a much shorter symbolism:

$$
y=<m, \alpha, \beta>\text {. }
$$

\section{3. The procedure for solving the problem of comparator identification when choosing} fuzzy numbers $(\boldsymbol{L}-\boldsymbol{R})$-type as input data

In accordance with the proposed general scheme for solving the problem of comparator identification, let's introduce a regression model (1) and a system of inequalities (4). However, unlike the previous one, all components in (4) are fuzzy numbers. Therefore, all the differences $y_{j}-y_{j+1}, j=1,2, \ldots, n-1$, are also fuzzy. Let's introduce a set of variables $\xi_{j}=y_{j}-y_{j+1}, j=1,2, \ldots, n-1$, and in accordance with the rules for performing arithmetic operations on fuzzy numbers of the $(L-R)$-type [11], let's calculate the membership functions of fuzzy numbers $\xi_{j}, j=1,2, \ldots, n-1$. Let's write down the corresponding summary of the rules for performing binary operations. Let's introduce a pair of fuzzy numbers:

$$
F_{1}=<m_{1}, \alpha_{1}, \beta_{1}>\text { and } F_{2}=<m_{2}, \alpha_{2}, \beta_{2}>\text {. }
$$

Then, when adding, let's obtain:

$$
\begin{gathered}
Z=F_{1}+F_{2}=<m_{Z}, \alpha_{Z}, \beta_{Z}> \\
m_{Z}=m_{1}+m_{2}, \quad \alpha_{Z}=\alpha_{1}+\alpha_{2}, \quad \beta_{Z}=\beta_{1}+\beta_{2} ;
\end{gathered}
$$

subtracting let's obtain:

$$
\begin{gathered}
Z=F_{1}-F_{2}=<m_{Z}, \alpha_{Z}, \beta_{Z}>, \\
m_{Z}=m_{1}-m_{2}, \quad \alpha_{Z}=\alpha_{1}+\beta_{2}, \quad \beta_{Z}=\beta_{1}+\alpha_{2} ;
\end{gathered}
$$


multiplying let's obtain:

$$
\begin{gathered}
Z=F_{1} \cdot F_{2}=<m_{Z}, \alpha_{Z}, \beta_{Z}>, \\
m_{Z}=m_{1} \cdot m_{2}, \alpha_{Z}=m_{1} \cdot \alpha_{2}+m_{2} \cdot \alpha_{1}-\alpha_{1} \cdot \alpha_{2}, \beta_{Z}=m_{1} \cdot \beta_{2}+m_{2} \cdot \beta_{1}+\beta_{1} \cdot \beta_{2} ;
\end{gathered}
$$

dividing let's obtain:

$$
\begin{gathered}
Z=F_{1} \cdot F_{2}=<m_{Z}, \alpha_{Z}, \beta_{Z}> \\
m_{Z}=\frac{m_{1}}{m_{2}}, \quad \alpha_{Z}=\frac{m_{2} \cdot \alpha_{1}+m_{1} \cdot \beta_{2}}{m_{2}\left(m_{2}+\beta_{2}\right)}, \quad \beta_{Z}=\frac{m_{1} \cdot \alpha_{2}+m_{2} \cdot \beta_{1}}{m_{2}\left(m_{2}-\alpha_{2}\right)} .
\end{gathered}
$$

Then

$$
\xi_{j}=y_{j}-y_{j+1}=x_{1}\left(F_{j 1}-F_{j+1,1}\right)+x_{2}\left(F_{j 2}-F_{j+1,2}\right)+\ldots+x_{m}\left(F_{j-1, m}-F_{j m}\right) .
$$

Because

$$
F_{j i}=<m_{j i}, \alpha_{j i}, \beta_{j i}>, \quad F_{j+1, i}=<m_{j+1, i}, \alpha_{j+1, i}, \beta_{j+1, i}>,
$$

then

$$
\begin{gathered}
F_{j i}-F_{j+1, i}=r_{j i}=<m_{r_{j i}}, \alpha_{r_{j i}}, \beta_{r_{i j}}> \\
m_{r_{j i}}=m_{j i}-m_{j+1, i}, \quad \alpha_{r_{j i}}=\alpha_{j i}+\beta_{j+1, i}, \quad \beta_{r_{i j}}=\beta_{j i}+\alpha_{j+1, i}, \\
j=1,2, \ldots, n-1, \quad i=1,2, \ldots, m .
\end{gathered}
$$

From relation (14), which determines the result before multiplying two fuzzy numbers, let's obtain formulas for the result of multiplying a fuzzy number by a crisp number.

Let's write a crisp number $x_{i}$ using the notation used for describing fuzzy numbers $(L-R)$-type: $x_{i}=\left\langle x_{i}, 0,0>\right.$. Then the fuzzy result of multiplying the crisp number $x_{i}$ by the fuzzy number $r_{j i}$ will be:

$$
x_{i} r_{j i}=\Delta_{j i}=<m_{\Delta_{i j}}, \alpha_{\Delta_{j i}}, \beta_{\Delta_{j i}}>\text {. }
$$

Then

$$
\begin{gathered}
\xi_{j}=\sum_{i=1}^{m} x_{i} r_{j i}=<m_{\xi_{j}}, \alpha_{\xi_{j}}, \beta_{\xi_{j}}> \\
m_{\xi_{j}}=\sum_{i=1}^{m} x_{i} m_{r_{j i}}, \quad \alpha_{\xi_{j}}=\sum_{i=1}^{m} x_{i}\left(\alpha_{j i}+\beta_{j+1, i}\right), \quad \beta_{\xi_{j}}=\sum_{i=1}^{m} x_{i}\left(\beta_{j i}+\alpha_{j+1, i}\right) .
\end{gathered}
$$

So,

$$
\begin{gathered}
\xi=\sum_{j=1}^{n-1} \xi_{j}=\sum_{j=1}^{n-1} \sum_{i=1}^{m} x_{i} r_{j i}=<m_{\xi}, \alpha_{\xi}, \beta_{\xi}>, \\
m_{\xi_{j}}=\sum_{j=1}^{n-1} m_{\xi_{j}}=\sum_{j=1}^{n-1} \sum_{i=1}^{m} x_{i} m_{r_{j i}}, \quad \alpha_{\xi}=\sum_{j=1}^{n-1} \alpha_{\xi_{j}}=\sum_{j=1}^{n-1} \sum_{i=1}^{m} x_{i}\left(\alpha_{j i}+\beta_{j+1, i}\right), \\
\beta_{\xi}=\sum_{j=1}^{n-1} \beta_{\xi_{j}}=\sum_{j=1}^{n-1} \sum_{i=1}^{m} x_{i}\left(\beta_{j i}+\alpha_{j+1, i}\right) .
\end{gathered}
$$


All analytical relationships required for the formation of a quality criterion for solving the problem of comparator identification in conditions of fuzzy initial data are obtained. The required set $\left(x_{1}, x_{2}, \ldots, x_{m}\right)$, satisfying the normalization condition (6), should be chosen so that all fuzzy numbers $\xi_{j}, j=1,2, \ldots, n-1$, are non-positive.

It is clear that the requirement of non-positiveness of the fuzzy numbers $\xi_{j}, j=1,2, \ldots, n-1$, can't be met if the fuzzy numbers $F_{j i}$ specifying the values of $\xi_{j}$ are defined on an infinite carrier. However, if these fuzzy numbers are given on a compact medium, then the problem can be solved. Let, for example, fuzzy numbers $F_{j i}$ have a triangular membership function:

$$
\mu\left(F_{j i}\right)=\left\{\begin{array}{l}
0, F_{j i}<m_{j i}-\alpha_{j i}, \\
\frac{F_{j i}-\left(m_{j i}-\alpha_{j i}\right)}{\alpha_{j i}}, m_{j i}-\alpha_{j i} \leq F_{j i}<m_{j i}, \\
\frac{\left(m_{j i}+\beta_{j i}\right)-F_{j i}}{\beta_{j i}}, m_{j i} \leq F_{j i}<m_{j i}+\beta_{j i}, \\
0, F_{j i}>m_{j i}+\beta_{j i} .
\end{array}\right.
$$

In this case, the maximum value of the fuzzy number $F_{j i}$ is equal to $m_{j i}+\beta_{j i}$, and the maximum value of the number $F_{j i}-F_{j+1}$ is equal to $m_{j i}-m_{j+1, i}+\beta_{j i}+\alpha_{j+1, i}$.

Then the maximum possible value $\xi_{j}$ on the set $\left(x_{1}, x_{2}, \ldots, x_{m}\right)$ is determined by the relation:

$$
\xi_{j \max }=\max _{x}\left[\sum_{i=1}^{m} x_{i}\left(m_{j i}-m_{j+1, i}+\beta_{j i}+\alpha_{j+1, i}\right)\right] .
$$

In this case, the problem can be formulated as follows: find a set $\left(x_{1}, x_{2}, \ldots, x_{m}\right)$ that satisfies the system of equations:

$$
\begin{gathered}
\sum_{i=1}^{m} x_{i}\left(m_{j i}-m_{j+1, i}+\beta_{j i}+\alpha_{j+1, i}\right)+x_{m+j}=0, \\
\sum_{i=1}^{m} x_{i}=1, \\
x_{i} \geq 0, \quad x_{m+j} \geq 0, \quad j=1,2, \ldots, n-1, \quad i=1,2, \ldots, m .
\end{gathered}
$$

Simultaneous fulfillment of conditions (19), (21) ensures the non-positiveness of $\xi_{j}$, $j=1,2, \ldots, n-1$. In the system of equations (19)-(21), the number of unknowns exceeds the number of equations. Therefore, as before, it is advisable to go over to the problem of minimizing a quadratic form.

In some cases, in accordance with the meaning of the identification problem, it is required that the inequalities $\xi_{j}<0, j=1,2, \ldots, n-1$ are satisfied as convincingly as possible. Then the condition for maximizing the linear form can be added to (19)-(21):

$$
L(x)=\sum_{i=1}^{n-1} x_{m+j} .
$$

In this case, problem (19)-(22) is transformed to a standard linear programming problem. Its solution is the desired solution to the comparator identification problem under conditions of fuzzy input data.

Practice shows that uncertainty models formed using standard fuzzy mathematics tools make it possible to obtain an acceptable solution to a larger number of emerging problems. However, in many real-life situations, the nature of uncertainty is more complex than usual. In particular, this situation arises when trying to describe the uncertainty of demand with a fuzzy number with a triangular membership function. It turns out that the values of the lower and upper boundaries of the 
uncertainty interval, as well as the modal value of this fuzzy number, based on the results of statistical processing of the initial data, can't be determined accurately. It seems natural to consider these values fuzzy. For a correct description of uncertainty in this case, it is advisable to use fuzzy numbers, the parameters of the membership functions of which are also fuzzy. The numbers obtained in this case were introduced by L. Zadeh in [10] and were called fuzzy numbers of type 2 (or fuzzy numbers of the second order, or bifuzzy numbers). Correct rules for performing arithmetic operations on second-order fuzzy numbers naturally generalize the rules proposed in [11]. Let's present those of them that will be used in the future for calculating the bi-fuzzy analogue of the objective function (19) in the problem of comparator identification. Let's introduce a pair of bifuzzy numbers $F_{1}$ and $F_{2}$ equal to:

$$
\begin{gathered}
F_{1}=<m_{1}, \alpha_{1}, \beta_{1}>, \quad F_{2}=<m_{2}, \alpha_{2}, \beta_{2}> \\
m_{1}=<m_{m_{1}}, \alpha_{m_{1}}, \beta_{m_{1}}>, \quad \alpha_{1}=<m_{\alpha_{1}}, \alpha_{\alpha_{1}}, \beta_{\alpha_{1}}>, \quad \beta_{1}=<m_{\beta_{1}}, \alpha_{\beta_{1}}, \beta_{\beta_{1}}> \\
m_{2}=<m_{m_{2}}, \alpha_{m_{2}}, \beta_{m_{2}}>, \quad \alpha_{2}=<m_{\alpha_{2}}, \alpha_{\alpha_{2}}, \beta_{\alpha_{2}}>, \quad \beta_{2}=<m_{\beta_{2}}, \alpha_{\beta_{2}}, \beta_{\beta_{2}}>.
\end{gathered}
$$

Then, in accordance with (14),

$$
\begin{gathered}
z=F_{1}-F_{2}=<m_{z}, \alpha_{z}, \beta_{z}> \\
m_{z}=m_{1}-m_{2}=<m_{m_{1}}, \alpha_{m_{1}}, \beta_{m_{1}}>-<m_{m_{2}}, \alpha_{m_{2}}, \beta_{m_{2}}>=<m_{m_{z}}, \alpha_{m_{z}}, \beta_{m_{z}}> \\
m_{m_{z}}=m_{m_{1}}-m_{m_{2}}, \quad \alpha_{m_{z}}=\alpha_{m_{1}}+\beta_{m_{2}}, \quad \beta_{m_{z}}=\beta_{m_{1}}+\alpha_{m_{2}}, \\
\alpha_{z}=\alpha_{1}+\beta_{2}=<m_{\alpha_{1}}, \alpha_{\alpha_{1}}, \beta_{\alpha_{1}}>+<m_{\beta_{2}}, \alpha_{\beta_{2}}, \beta_{\beta_{2}}>=<m_{\alpha_{2}}, \alpha_{\alpha_{2}}, \beta_{\alpha_{2}}> \\
m_{\alpha_{z}}=m_{\alpha_{1}}-m_{\beta_{2}}, \quad \alpha_{\alpha_{z}}=\alpha_{\alpha_{1}}+\alpha_{\beta_{2}}, \quad \beta_{\alpha_{z}}=\beta_{\alpha_{1}}+\beta_{\beta_{2}} ; \\
\beta_{z}=\beta_{1}+\alpha_{2}=<m_{\beta_{1}}, \alpha_{\beta_{1}}, \beta_{\beta_{1}}>+<m_{\alpha_{2}}, \alpha_{\alpha_{2}}, \beta_{\alpha_{2}}>=<m_{\beta_{z}}, \alpha_{\beta_{z}}, \beta_{\beta_{z}}> \\
m_{\beta_{z}}=m_{\beta_{1}}-m_{\alpha_{2}}, \quad \alpha_{\beta_{z}}=\alpha_{\beta_{1}}+\alpha_{\alpha_{2}}, \quad \beta_{\beta_{z}}=\beta_{\beta_{1}}+\beta_{\alpha_{2}} .
\end{gathered}
$$

The obtained relations set the parameters of the membership function of the bifuzzy number $\xi_{1}=F_{1}-F_{2}$. The relations for the binomial numbers $\xi_{j}=F_{j}-F_{j+1}, j=1,2, \ldots, n-1$. Let's write the result of multiplying the number $\xi_{1}$ by the constant $x_{1}$. Using (14), it is easy to show that when multiplying a bifuzzy number by a constant, a bifuzzy number will be obtained, all the parameters of the membership functions of which will be equal to the corresponding parameters of the bifuzzy factor multiplied by this constant. Then:

$$
\begin{gathered}
\Delta_{1}=\xi_{1} x_{1}=<m_{\Delta}, \alpha_{\Delta}, \beta_{\Delta}> \\
m_{\Delta}=<m_{m_{1}} x_{1}, \alpha_{m_{1}} x_{1}, \beta_{m_{1}} x_{1}>; \alpha_{\Delta}=<m_{\alpha_{1}} x_{1}, \alpha_{\alpha_{1}} x_{1}, \beta_{\alpha_{1}} x_{1}>; \beta_{\Delta}=<m_{\beta_{1}} x_{1}, \alpha_{\beta_{1}} x_{1}, \beta_{\beta_{1}} x_{1}>.
\end{gathered}
$$

Finally, let's obtain a relation for calculating the parameters of the sum of bifuzzy numbers $\Delta_{i}=\xi_{i} x_{i}$. Wherein

Because,

$$
\Delta=\sum_{i=1}^{m} \Delta_{1}=\sum_{i=1}^{m} \xi_{1} x_{1}=<m_{\Delta}, \alpha_{\Delta}, \beta_{\Delta}>.
$$

$$
\begin{gathered}
\Delta_{i}=\xi_{i} x_{i}=<m_{\Delta_{i}}, \alpha_{\Delta_{i}}, \beta_{\Delta_{i}}>, \\
m_{\Delta_{i}}=<m_{m_{\Delta_{i}}} x_{i}, \alpha_{m_{\Delta_{i}}} x_{i}, \beta_{m_{\Delta_{i}}} x_{i}>, \quad \alpha_{\Delta_{i}}=<m_{\alpha_{\Delta_{i}}} x_{i}, \alpha_{\alpha_{\Delta_{i}}} x_{i}, \beta_{\alpha_{\Delta_{i}}} x_{i}>, \beta_{\Delta_{i}}=<m_{\beta_{\Delta_{i}}} x_{i}, \alpha_{\beta_{\Delta_{i}}} x_{i}, \beta_{\beta_{\Delta_{i}}} x_{i}>,
\end{gathered}
$$


So,

$$
\begin{aligned}
& m_{\Delta}=<\sum_{i=1}^{m} m_{m_{\Delta_{i}}} x_{i}, \sum_{i=1}^{m} \alpha_{m_{\Delta_{i}}} x_{i}, \sum_{i=1}^{m} \beta_{m_{\Delta_{i}}} x_{i}>, \\
& \alpha_{\Delta}=<\sum_{i=1}^{m} m_{\alpha_{\Delta_{i}}} x_{i}, \sum_{i=1}^{m} \alpha_{\alpha_{\Delta_{i}}} x_{i}, \sum_{i=1}^{m} \beta_{\alpha_{\Delta_{i}}} x_{i}>, \\
& \beta_{\Delta}=<\sum_{i=1}^{m} m_{\beta_{\Delta_{i}}} x_{i}, \sum_{i=1}^{m} \alpha_{\beta_{\Delta_{i}}} x_{i}, \sum_{i=1}^{m} \beta_{\beta_{\Delta_{i}}} x_{i}>.
\end{aligned}
$$

Now, similarly to the previous one, let's write down the maximum value $\xi_{j}$.

$$
\begin{gathered}
m_{\Delta}=<\sum_{i=1}^{m} m_{m_{\Delta_{i}}} x_{i}, \sum_{i=1}^{m} \alpha_{m_{\Delta_{i}}} x_{i}, \sum_{i=1}^{m} \beta_{m_{\Delta_{i}}} x_{i}> \\
\xi_{j \max }=\max _{x}\left[\sum_{i=1}^{m} m_{m_{\Delta_{i}}} x_{i}+\sum_{i=1}^{m} \beta_{m_{\Delta_{i}}} x_{i}+\sum_{i=1}^{m} m_{\beta_{\Delta_{i}}} x_{i}+\sum_{i=1}^{m} \beta_{\beta_{\Delta_{i}}} x_{i}\right]= \\
=\max _{x}\left(m_{m_{\Delta_{i}}}+\beta_{m_{\Delta_{i}}}+m_{\beta_{\Delta_{i}}}+\beta_{\beta_{\Delta_{i}}}\right) x_{i} .
\end{gathered}
$$

Then the problem is formed as follows: find a set $x_{i}, i=1,2, \ldots, m$, satisfying the system of equations:

$$
\begin{gathered}
\sum_{i=1}^{m} x_{i}\left(m_{m_{\Delta_{i}}}+\beta_{m_{\Delta_{i}}}+m_{\beta_{\Delta_{i}}}+\beta_{\beta_{\Delta_{i}}}\right)+x_{m+j}=0, \\
\sum_{i=1}^{m} x_{i}=1, \quad x_{i} \geq 0, \quad x_{m+j} \geq 0, \quad j=1,2, \ldots, n-1, \quad i=1,2, \ldots, m .
\end{gathered}
$$

It is clear that the joint satisfaction of conditions (23), (24) ensures the non-positiveness of $\xi_{j}, j=1,2, \ldots, n-1$, as required. Just as before, if for a specific identification problem it is necessary that the non-positiveness of $\xi_{j}$ be maximally convincing, then the requirement of maximizing the linear form (22) is added to (23), (24), which reduces to a linear programming problem. It is clear that the solution obtained in this case is better than the previous one, since it is more stable with respect to possible errors in estimating the numerical values of the parameters of the membership functions of fuzzy initial data.

\section{Discussion of the results of solving the problem of comparator identification in conditions of fuzzy initial data}

Comparative identification is a method for solving the problem of regression analysis, the purpose of which is to establish the dependence of the explained variable on the explanatory variables (factors) that affect the explained variable. The comparator identification problem and the method for its solution have been developed and applied in cases when during the collection of initial data it is not possible to measure the values of the variable being explained, but the results of experiments can be ranked. The paper considers a variant of the comparator identification problem, which is important for practice, when the measurement of the values of the factors in each experiment is not clearly specified. Such problems arise in various practical applications: to increase the efficiency of vocational guidance activities of educational institutions [12], to predict the emergence and development of social processes, including negative trends in society [13], in the management of industrial waste [14] and technological processes in industry [15], construction [16]. The expediency of applying the proposed solutions in these areas is justified by the complexity, and sometimes the impossibility of measuring and evaluating the variables that describe the processes. If, in addition to the resulting uncertainties caused by these objective facts, take into account the uncertainties caused by the need to attract expert opinion and obtain appropriate 
expert assessments, there is reason to assert the effectiveness of comparator identification in conditions of fuzzy description. The latter is especially important in areas with an increased level of requirements for the safety of the operation and operation of facilities [17-19]. Taking into account the fact that the solution of problems arising in these applications by traditional methods of regression analysis is impossible, the method proposed in this work, which reduces the problem to solving the arising system of linear algebraic equations, the parameters of which are given indistinctly, is an effective mathematical apparatus. For the case when all fuzzy parameters are specified on a compact carrier, the method provides a solution to a fuzzy system of linear algebraic equations. The proposed computational procedure has been developed and can be applied in a situation where the uncertainty in the value of factors is described in terms of second-order fuzzy numbers. The paper also considered a possible enhancement of the method, which consists in the following. From the set of feasible options for solving the problem, the most stable is selected with respect to possible errors in setting the parameters of the membership functions of fuzzy initial data.

A possible direction for further research is the development of a method for solving the comparator identification problem for the case when the initial data are specified inaccurately [20]. To solve the problem, it is possible to use the technologies proposed in [21, 22].

\section{Conclusions}

1. The correct formulation of the comparator identification problem is formulated for the case when the initial data are given in terms of fuzzy mathematics. In this case, to describe the membership function of the corresponding fuzzy numbers, functions $(L-R)$-type are selected.

2. All analytical relationships required for the formation of a quality criterion for solving the problem of comparator identification in conditions of fuzzy initial data have been obtained. As a result, a criterion for the effectiveness of the solution is proposed, based on the calculation of membership functions of the results of experiments, and the transformation of the problem to a standard problem of linear programming is shown. The solution to the latter is what is sought for comparator identification under conditions of fuzzy initial data.

3. A procedure for solving the problem of comparator identification is proposed, when the initial data are fuzzy numbers $(L-R)$-type. The solution obtained by its implementation is more stable with respect to possible errors in estimating the numerical values of the parameters of the membership functions of fuzzy initial data.

\section{References}

[1] Vuchkov, I., Bachdzhieva, L., Solakov, B. (1987). Prikladnoy lineyniy regressionnyy analiz. Moscow: Finansy i statistika, 239.

[2] Rao, J. N. K., Subrahmaniam, K. (1971). Combining Independent Estimators and Estimation in Linear Regression with Unequal Variances. Biometrics, 27 (4), 971. doi: https://doi.org/10.2307/2528832

[3] Petrov, K. E., Kryuchkovskiy, V. V. (2009). Komparatornaya strukturno-parametricheskaya identifikatsiya modeley skalyarnogo mnogofaktornogo otsenivaniya. Kherson: Oldi-plyus, 294.

[4] Dotsenko, N. V., Kosenko, N. V. (2012). Comparator authentication of parameters of model of multifactor evaluation. Systemy upravlinnia, navihatsiyi ta zviazku, 2 (1 (21)), 140-143

[5] Bondarenko, M. F., Shabanov-Kushnarenko, Yu. P., Sharonova, N. V. (2010). The ideas algebra interpretations. Bionics of Intelligence, 2 (73), 74-86.

[6] Petrov, E., Petrov, K. (2014). Komparatornaya identifikatsiya modeley mnogofaktornogo otsenivaniya. Saarbrucken: Palmarium Academic Publishing, 224.

[7] Suprun, T. S., Shabanov-Kushnarenko, S. Y. (2014). Isomorphism of predicate model comparator identification. Radio Electronic, Computer Science, Control, 14-24.

[8] Raskin, L. G., Seraja, O. W. (2015). Information problems of canonical regression analysis. Systemy obrobky informatsiyi, 10, $230-234$

[9] Gantmaher, F. R. (2004). Teoriya matrits. Moscow: Fizmatlit, 560.

[10] Zadeh, L. A. (1965). Fuzzy sets. Information and Control, 8 (3), 338-353. doi: https://doi.org/10.1016/s0019-9958(65)90241-x

[11] Raskin, L., Sira, O. (2020). Performing arithmetic operations over the (L-R)-type fuzzy numbers. Eastern-European Journal of Enterprise Technologies, 3 (4 (105)), 6-11. doi: https://doi.org/10.15587/1729-4061.2020.203590 
[12] Yeremenko, B., Ryabchun, Y., Ploska, G. (2018). The introduction of intellectual system for evaluating professional abilities of applicants into the activities of educational institutions. Technology audit and production reserves, 6 (2 (44)), $22-26$. doi: https://doi.org/10.15587/2312-8372.2018.149680

[13] Mulesa, O. (2015). Adaptation of fuzzy c-means method for determination the structure of social groups. Technology Audit and Production Reserves, 2 (2 (22)), 73-76. doi: https://doi.org/10.15587/2312-8372.2015.41014

[14] Baranova, A., Samoilenko, N., Pitak, I. (2020). Forecasting of formation of pharmaceutical glass waste taking into account the COVID-19 database. ScienceRise, 4, 46-52. doi: https://doi.org/10.21303/2313-8416.2020.001392

[15] Domin, D. (2013). Artificial orthogonalization in searching of optimal control of technological processes under uncertainty conditions. Eastern-European Journal of Enterprise Technologies, 5 (9 (65)), 45-53. doi: https://doi.org/10.15587/ 1729-4061.2013.18452

[16] Popyk, N. (2014). Ontological approach and fuzzy modeling to describe objects of living environment. Technology Audit and Production Reserves, 6 (4 (20)), 7-9. doi: https://doi.org/10.15587/2312-8372.2014.32875

[17] Kartavykh, S., Komandyrov, O., Kulikov, P., Ploskyi, V., Poltorachenko, N., Terenchuk, S. (2020). Adaptation of fuzzy inference system to solve assessment problems of technical condition of construction objects. Technology Audit and Production Reserves, 3 (2 (53)), 52-55. doi: https://doi.org/10.15587/2706-5448.2020.205364

[18] Grigorovskiy, P., Terentyev, O., Mikautadze, R. (2017). Development of the technique of expert assessment in the diagnosis of the technical condition of buildings. Technology Audit and Production Reserves, 2 (2 (40)), 10-15. doi: https://doi.org/ $10.15587 / 2312-8372.2018 .128548$

[19] Pasko, R., Terenchuk, S. (2020). The use of neuro-fuzzy models in expert support systems for forensic building-technical expertise. ScienceRise, 2, 10-18. doi: https://doi.org/10.21303/2313-8416.2020.001278

[20] Pawlak, Z. (1982). Rough sets. International Journal of Computer \& Information Sciences, 11 (5), 341-356. doi: https://doi.org/ 10.1007/bf01001956

[21] Raskin, L., Sira, O. (2016). Fuzzy models of rough mathematics. Eastern-European Journal of Enterprise Technologies, 6 (4 (84)), 53-60. doi: https://doi.org/10.15587/1729-4061.2016.86739

[22] Raskin, L., Sira, O. (2016). Method of solving fuzzy problems of mathematical programming. Eastern-European Journal of Enterprise Technologies, 5 (4 (83)), 23-28. doi: https://doi.org/10.15587/1729-4061.2016.81292

Received date 20.05.2020

Accepted date 15.01.2021

Published date 29.01.2021
(C) The Author(s) 2021

This is an open access article under the CC BY license (http://creativecommons.org/licenses/by/4.0). 